\title{
El engagement de participantes en MOOC (Massive Open Online Courses): Análisis del diseño instruccional y elementos alternativos
}

\author{
Incidence of instructional design and alternative elements in the \\ engagement of participants in MOOC
}

\author{
Bárbara Castillo-Abdul \\ Universidad Rey Juan Carlos, ESAI Business School- Universidad Espíritu Santo \\ Mónica Bonilla-Del-Río \\ Sabina Civila \\ Universidad de Huelva
}

\begin{abstract}
Resumen
Los MOOC (Massive Open Online Courses) han significado una oportunidad de formación y emprendimiento en el escenario educativo. La presente investigación aborda un caso de estudio, cuyo objetivo es conocer e identificar las características que inciden en la motivación de los participantes de las vídeo-lecciones que conforman tres cursos ofertados en las plataformas MiríadaX, Edx y ECO: E-learning, Communication y Open-Data". El criterio de selección de estas plataformas se basó en su popularidad, relevancia en el contexto actual y enfoque en la estrategia de promoción de contenidos en el entorno digital. Se concluye que el diseño y las características analizadas de los MOOC inciden en el nivel de engagement de los participantes. En los casos presentados, asimismo, el diseño tradicional educativo de clases virtuales manifiesta carencias de elementos alternativos de gamificación, networking, lo que podría generar un bajo nivel de interés y compromiso por parte de los participantes.
\end{abstract}

Palabras clave: MOOC; Innovación; Educación; Marketing digital

\begin{abstract}
The MOOC (Massive Open Online Courses) have meant an opportunity for training and entrepreneurship in the educational arena. The present research addresses a case study, which aims to know and identify the characteristics that affect the motivation of the participants of the video-lessons that make up three courses offered on the platforms MyriadX, Edx, and ECO: E-learning, Communication and OpenData. The selection criteria of these platforms were based on their popularity, relevance in the current context, and focused on the strategy of promoting content in the digital environment. It is concluded that the design and characteristics analyzed of the MOOC affect the level of engagement of the participants. Furthermore, in the cases presented the traditional educational map of virtual classrooms shows a lack of alternative elements of gamification, networking, which could generate a low level of interest and commitment by the participants.
\end{abstract}

Keywords: MOOC; Innovation; Education; Digital marketing 


\section{INTRODUCCIÓN: LOS MOOC Y LAS COMPETENCIAS DIGITALES}

Desde el nacimiento en 2008de los MOOC (Massive Open Online Courses), la comunidad científica ha tenido gran interés en su estudio, debido a su gran versatilidad. Estos cursos se han conformado como una alternativa de educación flexible y adaptada a las características de la sociedad actual, influyendo en las opciones ofertadas por las universidades que, impulsadas por la necesidad de conciliación a esta nueva realidad, apuestan por el diseño y promoción de los recursos para la formación en línea, lo que favorece la evolución del e-learning (Castaño-Garrido et al., 2015). Sin embargo, las tasas de finalización de este tipo de recursos son relativamente bajas, un cierto fracaso que se ha atribuido al diseño monolítico del propio curso, que no permite desarrollar características específicas para subpoblaciones con requerimientos diferentes a los de la mayoría de alumnos (Kizilcec et al., 2013).

Los MOOC han sido definidos como:

"Cursos online diseñados para un gran número de participantes, a los cuales puede acceder cualquier persona desde cualquier lugar, siempre y cuando se cuente con conexión a Internet, son gratis para todos y todas y ofrece la experiencia de un aprendizaje online gratuito.” (Jansen \& Goes-Daniels, 2015, p. 11).

Los cursos consisten en breves vídeos con contenido relacionado con una temática concreta, que suelen incluir ejercicios prácticos, correcciones automáticas y feedback a través de cuestionarios. Además, ofrecen grupos de discusión, foros, blogs y wikis (Glance et al., 2013). Los MOOC han democratizado el acceso al conocimiento y a los contenidos formativos gracias a sus características de gratuidad, ubicuidad y heterogeneidad y a sus amplias posibilidades de innovación en modelos instruccionales, nuevas arquitecturas de la gestión del conocimiento y su enfoque de life-long learning, lo que conlleva a entenderlos como un nuevo paradigma pedagógico (Romero-Rodríguez et al., 2019).

La decisión de inscribirse en un MOOC depende, en gran medida, de cómo los participantes evalúan sus conocimientos preexistentes sobre la materia, su disponibilidad horaria, sus recursos y competencias digitales y sus capacidades de autorregulación (Zhou, 2016). Por su parte, White et al. (2014) explican que las tres razones principales para escoger un MOOC son (en este orden): su gratuidad, el deseo de actualizar conocimientos y el interés por la materia; mientras que Zheng et al. (2015) identifican cuatro tipos de motivaciones para inscribirse: satisfacer necesidades actuales, prepararse para el futuro, satisfacer alguna curiosidad y conocer personas del propio ámbito académico. 
Para la inscripción en este tipo de cursos es necesario tener ciertas competencias digitales, desde las capacidades o habilidades de los usuarios para procesar, relacionar, buscar y expresar la información hasta aquellas de pensar de modo más fragmentado, visual, interactivo y rápido (Pérez-Rodríguez \& Delgado-Ponce, 2012). La rapidez de cambios del ecosistema digital, que ha pasado de web estática a redes sociales interactivas, blogs, vídeoblogs, cursos en línea (web 2.0) e inmersión en realidad virtual y aumentada, MOOC y weareables (web 3.0), han supuesto en cierta medida que los usuarios aprendan sobre estas nuevas plataformas de manera autodidacta (Romero-Rodríguez et al., 2016). Teniendo en cuenta el rápido avance de la tecnología, quien tenía competencias digitales hace unos años puede que carezca de ellas ahora. Además, si a esto se le suma la dificultad de accesibilidad digital de algunas personas podría ser más fácil comprender el fracaso de los MOOC que se ha mencionado previamente.

La competencia digital, caracterizada por su carácter evolutivo, no lineal y complejo (Padilla-Hernández et al., 2019), puede resumirse en cinco áreas concretas (European Comission, 2019):

- Alfabetización informacional y análisis de datos: Comprende las habilidades para localizar y recuperar datos e información digital, valorar la relevancia de la fuente y la pertinencia de los contenidos, así como para almacenarlos, organizarlos y gestionarlos eficientemente.

- Creación de contenido digital: Está conformada por las habilidades para crear y editar contenido mejorando e integrando los nuevos conocimientos a la información existente, respetando las licencias y los derechos de autor.

- Comunicación y colaboración: Abarca las destrezas de interacción a través de las tecnologías, atendiendo a la diversidad generacional y cultural, así como las habilidades para participar en sociedad haciendo uso de los servicios digitales públicos y privados. También engloba la competencia para gestionar la propia identidad y reputación digital.

- Seguridad: Incluye el conjunto de habilidades para proteger los dispositivos, los contenidos, los datos personales y la privacidad en entornos virtuales, lo que implica la protección de la salud física y psicológica, la utilización adecuada de las tecnologías para el bienestar y la inclusión social, siendo conscientes de su impacto ambiental y su uso.

- Resolución de problemas: Hace referencia a la competencia para identificar y resolver las necesidades y dificultades en entornos virtuales. Además, 
engloba las destrezas para usar herramientas digitales que permitan innovar procesos, productos y mantenerse al día.

En esta línea, surge la necesidad de desarrollar la competencia digital de la ciudadanía en un doble sentido: a) formación específica para evitar que su desarrollo recaiga únicamente en un aprendizaje individual de tipo intuitivo -a imitación de aprendizaje de cualquier software o entorno digital comercial-; b) un entorno que ayude al usuario a implicarse en el desarrollo de su propio aprendizaje. En las líneas que siguen se pone el foco en b).

\section{INNOVACIÓN EN EL DESARROLLO DE LOS MOOC}

La integración de estrategias educativas innovadoras puede impactar en el desarrollo y éxito de un MOOC. A continuación, se presentan tres modelos de enseñanza y aprendizaje que constituyen marcos para adaptar estos procesos al entorno digital: gamificación, el aprendizaje basado en retos y el aprendizaje invertido (Valenzuela-Arviszu et al., 2019).

En primer lugar, la gamificación consiste en la utilización de interfaces características de vídeojuegos, con el objetivo de aumentar la fidelidad y el interés en el curso (Teixes, 2014). En segundo lugar, el aprendizaje basado en retos tiene la finalidad de hacer frente a las enseñanzas tradicionales, mediante el trabajo colaborativo y práctico con diferentes grupos multidisciplinares, lo que permite que los alumnos construyan su conocimiento desde una perspectiva crítica y realista (Apple Inc, 2011). Y, por último, el aprendizaje invertido, definido por Ramírez y Ramírez (2016) como un cambio en el proceso de nociones, centra la atención de los estudiantes y de los profesores en un ambiente no convencional. En este tipo de enseñanza las clases presenciales se utilizan para actividades prácticas, mientras que el contenido teórico es analizado de forma telemática. Estos planteamientos, favorecen una innovación abierta, colaborativa y multidisciplinar. Es decir, optimizan los esfuerzos aportando beneficios compartidos y fomentan la integración de diversas disciplinas. lo que redunda en la diversificación de los recursos y la mejora de habilidades.

Las tecnologías han cambiado el mundo y, por supuesto, la forma de aprendizaje también. La educación a través de plataformas online se ha convertido en un escenario de innovación y están en la búsqueda de nuevos modelos pedagógicos que se adapten a este tipo de enseñanzas y que ayuden al usuario a finalizar los cursos. El entorno digital y sus herramientas son muy importantes para desarrollar una comunicación de valor entre el docente y el alumno, entendiendo que la calidad 
de los recursos disponibles favorece la relación entre emisor y receptor (García, 2001).

Sin duda, los cursos masivos exigen un cambio en la forma de enseñanza del profesorado, ya que la enseñanza tradicional no tiene éxito en entornos digitales y la forma en la que los alumnos acceden al contenido es muy variada. Por lo tanto, se presenta un reto mayor para el docente, quien deberá preparar materiales digitales adaptados, que mejoren la calidad de interacción entre docentes y alumnado (Mcgowan \& Hanna, 2016). De acuerdo con Gallego y Gutiérrez (2011), el modelo educativo tradicional sigue latente en muchos de los MOOC, por lo que se han ido desarrollando nuevas estrategias para acercarse al alumnado utilizando entornos digitales originales y motivadores.

Uno de los principales problemas para el profesorado es su escaso conocimiento en TIC y los nuevos modelos de educación, lo que dificulta el desarrollo de metodologías didácticas innovadoras. En este sentido, muchos docentes se graban como si estuvieran impartiendo lecciones en clases presenciales, convirtiendo los MOOC en una serie de vídeos cortos o, como indica Piscitelli (2015), en un «boulevard de clips». Este hecho provoca que el alumno se salte contenido del curso y en muchas ocasiones no lo finalice (Atapattu \& Falkner, 2017).

La variedad de vídeo-lecciones que se pueden encontrar en los MOOC ha sido catalogada por Hansch et al. (2015) en tres grandes grupos: a) Talking Head, aquellos vídeos en los que hay una persona frente a la cámara explicando una lección, lo que se conoce como «busto parlante»; b) Picture in picture, imágenes con voz en off, que pueden ser la interfaz de un programa, capturas de pantallas o animaciones; c) entrevistas, tutoriales o vídeos de tipo magistral con la inclusión de presentaciones de Power Point. Los distintos tipos de vídeos, la duración recomendada para que los alumnos tengan motivación e interés, el tipo de tecnología que se utiliza para la grabación y los recursos digitales novedosos ofrecidos hacen que sea relevante comprender el éxito de cada uno de ellos y explorar en qué medida unos favorecen más el aprendizaje que otros.

Las plataformas de gestión de aprendizajes, más conocidas como Learning Management System (LMS), entre las que se incluyen los escenarios como foros virtuales, aplicaciones para trabajo colaborativo a distancia y otras herramientas, siguen transformando el diseño instruccional de cursos y dejan el reto constante de innovar hacia la construcción de ambientes de aprendizaje enriquecidos en donde la tecnología es un medio y no un fin en sí mismo. 


\section{TENDENCIAS MÁS RELEVANTES Y DESAFÍOS DE LOS MOOC}

Hablar de tendencias en el aprendizaje digital es bastante difícil, pues se encuentra en continuo cambio, aunque, de acuerdo con Manotas-Salcedo (2019), tendencias como el OCW (Open Course Ware) y el conectivismo son pilares claves en el desarrollo de los MOOC, ya que influyeron en los inicios de los primeros cursos masivos online, sirviendo como referentes en cuanto a la educación a distancia a través de las TIC y la promoción del acceso libre al conocimiento. A su vez, los MOOC están potenciando el blended learning, sirviendo de ejemplo para modelos que plantean ambientes educativos híbridos que combinan la presencialidad y la virtualidad (Manotas-Salcedo, 2019). Estos recursos, utilizados de forma correcta, promueven un rol mucho más activo del estudiante durante la realización de estos cursos, donde el docente aporta las herramientas necesarias para que se desarrollen opiniones críticas y conocimientos.

Para ello, el profesorado debe adaptarse a las nuevas tendencias y proponer actividades más dinámicas, fomentando la colaboración con el alumnado y centrando la evaluación en el proceso más que en el resultado (Vázquez-Cano et al., 2013). A pesar de los inequívocos aspectos positivos que conllevan recursos como Prezi o Power Ponint para organizar sesiones magistrales, su abuso puede constituir un obstáculo en la implicación de los alumnos por lo que el empleo de otros recursos podría ser clave a la hora de plantear este tipo de cursos online (Cabero $\&$ Gisbert, 2005), con el fin de favorecer precisamente un proceso de enseñanzaaprendizaje más colaborativo y constructivista. En el caso de una clase presencial, el profesorado puede captar la atención de los alumnos mediante la proyección de vídeos. En cambio, en la enseñanza digital, el vídeo debe enmarcar claramente un objetivo y resultado de aprendizaje en sí mismo porque este difícilmente se podría apoyar en una explicación posterior del docente.

En este contexto, las iniciativas para convertirse en tendencia están en continuo crecimiento. En este sentido, se han desarrollado diferentes tipologías de cursos (Gómez-Galán \& Pérez-Parras, 2017), como el cMOOC, el XMOOC y el tMOOC. El primero es un modelo que busca el aprendizaje a través de las interacciones entre estudiantes, centrado en el aprendizaje de red a través de teorías conectivistas.; el segundo tipo no conectivista se centra en el contenido que ofrece la página web del curso apoyándose en formatos tradicionales; finalmente, el tMOOC recoge tanto los aspectos del primero como segundo.

Uno de los mayores desafíos a los que se enfrenta la educación digital es la permanencia del alumnado durante todo el curso, de ahí la necesidad de generar 
cambios en la infraestructura tecnológica, creando todo tipo de herramientas y entornos virtuales que favorezcan su implicación y ayuden a superar los problemas derivados de lo que Vázquez-Cano et al. (2013) denominan "principio de masividad" y "falta de individualización".

Como consecuencia, autores como Aguaded y Medina-Salguero (2015) plantean dudas sobre si el sustento pedagógico de estos cursos garantiza el aprendizaje. Reeves (2006) sostiene que el éxito de aprendizaje de estos cursos masivos se encuentra en la concordancia entre 8 factores: resultados de aprendizaje, contenido, diseño de la interfaz, actividades, acceso a las TIC, rol del profesor, rol del estudiante y sistema de evaluación. Por tanto, el enfoque de los MOOC debe centrarse en el aprendizaje y no en dictar una clase como si del formato presencial se tratara. El docente debe diseñar ambientes de aprendizaje, construir experiencias y facilitar procesos.

El objetivo de este estudio es conocer e identificar las características que pueden incidir en la motivación de los participantes. Para ello, exploramos las vídeo-lecciones que conforman tres cursos ofertados en las plataformas MiríadaX, Edx y ECO: E-learning, Communication, Open-Data”.

\section{Metodología}

Este trabajo toma como referencia investigaciones realizadas previamente desde el enfoque cualitativo para analizar propuestas de formación en línea (Calvo-Salvador et al., 2016), en este caso específico, a través de un estudio de caso relativo a los MOOC. La selección es, por lo tanto, doble, puesto que se eligieron, por un lado, las plataformas online para cursar MOOC y, por otro, unos cursos concretos desarrollados en dichas plataformas. El criterio de selección de las plataformas se basó en su popularidad y relevancia en el contexto actual (Martín-Padilla, 2016), mientras que para los cursos se realizó una búsqueda mediante las siguientes palabras clave: "ciencias sociales", "comunicación digital" que derivó en la elección de tres cursos. El enfoque central de esos cursos es la estrategia en la promoción de contenidos en el entorno digital (Tabla1) a partir de un muestreo intencional no probabilístico en el cual se ha seleccionado la muestra basando la elección en un juicio subjetivo, como el más conveniente para la investigación planteada, en lugar de hacer la selección al azar (Otzen \& Manterola, 2017). Por tanto, para esta última selección, los MOOC debían cumplir, además, los siguientes requisitos: estar abiertos para la inscripción, estar ofertados en castellano, ser gratuitos, no necesitar de tutorización y no exigir requisitos específicos de acceso. 
Tabla 1. Características de los MOOC seleccionados

\begin{tabular}{llll}
\hline \multicolumn{1}{c}{ Título } & Plataforma & \multicolumn{1}{c}{ Institución } & \multicolumn{1}{c}{ Duración } \\
\hline $\begin{array}{l}\text { Introducción al Marketing Digital y } \\
\begin{array}{l}\text { Comercio Electrónico para Empren- } \\
\text { dedores y Empresarios }\end{array}\end{array}$ & MiríadaX & $\begin{array}{l}\text { Universidad Nacional } \\
\text { Abierta y a Distancia } \\
\text { (UNAD, Colombia) }\end{array}$ & $\begin{array}{l}4 \text { semanas } \\
\text { (12 horas a la } \\
\text { semana) }\end{array}$ \\
$\begin{array}{l}\text { Entrepreneurship 101: ¿Quién es tu } \\
\text { cliente? }\end{array}$ & Edx & $\begin{array}{l}\text { MITx (Massachusetts } \\
\text { Institute of Technol- } \\
\text { ogy, EEUU) }\end{array}$ & $\begin{array}{l}\text { 6 semanas (4 } \\
\text { horas a la se- } \\
\text { mana) }\end{array}$ \\
$\begin{array}{l}\text { Despertar la mirada: Introducción a } \\
\text { la lectura crítica de la imagen }\end{array}$ & ECO & No se especifica & $\begin{array}{l}\text { 4 semanas (5 } \\
\text { horas a la se- } \\
\text { mana) }\end{array}$ \\
\hline
\end{tabular}

Tras la selección de los cursos, se procedió a la realización del análisis de contenido. Esta técnica, de acuerdo con Igartua (2006), es adecuada y se emplea en aquellos estudios que requieren una aproximación científica al análisis de los mensajes, con el fin de lograr la comprensión de su origen o finalidad, una descripción precisa de sus características, estructura y componentes, así como la obtención de datos acerca de su flujo y la inferencia de su evolución e impacto.

Por su parte, según Piñuel-Raigada (2002), el análisis de contenido consiste en el "conjunto de procedimientos interpretativos de productos comunicativos (mensajes, textos o discursos) que proceden de procesos singulares de comunicación previamente registrados” (p. 2). Su finalidad sería la elaboración y procesamiento de información sustancial para conocer las condiciones en las que se han desarrollado o se podrían replicar en casos posteriores. De este modo, siguiendo con la fundamentación de este autor, la técnica de medida empleada en este estudio se engloba dentro de la perspectiva cualitativa, a partir del uso de lógicas que se centran en la comparación de categorías.

Para ello, se realizó la adaptación de un instrumento validado por expertos (Manotas-Salcedo et al., 2019) y aplicado en estudios anteriores en la plataforma MiriadaX (Manotas-Salcedo et al., 2018). La novedad del presente estudio consiste en la aplicación del instrumento para realizar una comparativa en distintas plataformas, con el fin de comprobar si existen similitudes y diferencias en los MOOC analizados. Dicho análisis está basado en cuatro categorías: inicio, desarrollo y cierre de la vídeo-lección (Tabla 2) y aspectos técnicos y de soporte narrativo audiovisual (Tabla 3), que incluyen el uso de recursos y la aplicación de principios de Storytelling (Manotas-Salcedo et al., 2018).

El análisis de los MOOC se realizó durante los meses de julio y agosto de 2020. 


\section{HERRAMIENTA DE ANÁLISIS}

Tabla 2. Inicio, desarrollo y cierre de la vídeo-lección

\begin{tabular}{|c|c|c|}
\hline Categorías & Acciones del docente & Observaciones \\
\hline \multirow{3}{*}{ Inicio } & $\begin{array}{l}\text { Comienza con una pre- } \\
\text { gunta a la audiencia. }\end{array}$ & $\begin{array}{l}\text { Se toma en consideración si la introducción al módulo } \\
\text { por parte del docente incluye una serie de interrogan- } \\
\text { tes. }\end{array}$ \\
\hline & $\begin{array}{l}\text { Plantea un reto a resol- } \\
\text { ver durante la vídeo lec- } \\
\text { ción. }\end{array}$ & $\begin{array}{l}\text { Se evalúan los desafíos planteados para acceder a la si- } \\
\text { guiente lección, más allá de los ejercicios y pruebas de } \\
\text { evaluación. }\end{array}$ \\
\hline & $\begin{array}{l}\text { Desglosa de manera } \\
\text { clara y organizada los } \\
\text { puntos que se desarro- } \\
\text { llarán en la vídeo-lec- } \\
\text { ción. }\end{array}$ & $\begin{array}{l}\text { Se analiza si el docente ofrece una breve explicación de } \\
\text { introducción sobre el módulo haciendo mención a cada } \\
\text { uno de los ejes o puntos que se desarrollarán en la lec- } \\
\text { ción. }\end{array}$ \\
\hline \multirow{4}{*}{ Desarrollo } & Usa giros de humor. & $\begin{array}{l}\text { Se tiene en cuenta si se usa el recurso humorístico y la } \\
\text { entonación para hacer énfasis durante el discurso, con } \\
\text { el fin de comprobar si estos rompen la monotonía del } \\
\text { estilo. }\end{array}$ \\
\hline & $\begin{array}{l}\text { Recurre a anécdotas } \\
\text { personales relacionadas } \\
\text { con el tema de la vídeo- } \\
\text { lección. }\end{array}$ & $\begin{array}{l}\text { Se pretende localizar si a través de anécdotas persona- } \\
\text { les el docente conecta con los alumnos para fomentar } \\
\text { la motivación, cercanía y continuidad del curso, así } \\
\text { como para comprobar si se recurre a estos recursos } \\
\text { para ejemplificar los contenidos que se desarrollan en el } \\
\text { MOOC. }\end{array}$ \\
\hline & $\begin{array}{l}\text { Incluye cambios de ritmo } \\
\text { en el vídeo. }\end{array}$ & $\begin{array}{l}\text { Se analiza si durante la exposición de los contenidos } \\
\text { que componen las vídeo-lecciones, se emplea una va- } \\
\text { riación en la producción y edición audiovisual. }\end{array}$ \\
\hline & $\begin{array}{l}\text { Incluye puntos de giro o } \\
\text { hechos inesperados }\end{array}$ & $\begin{array}{l}\text { Se valora si existen acontecimientos que causen sor- } \\
\text { presa en el estudiante o que inicialmente no estuvieran } \\
\text { previstos. }\end{array}$ \\
\hline \multirow{3}{*}{ Cierre } & $\begin{array}{l}\text { Establece conclusiones } \\
\text { de acuerdo con lo plan- } \\
\text { teado en el inicio de la } \\
\text { vídeo-lección. }\end{array}$ & $\begin{array}{l}\text { Se tiene en cuenta la presentación de las consideracio- } \\
\text { nes finales y conclusiones en relación con los conteni- } \\
\text { dos tratados en la vídeo-lección. }\end{array}$ \\
\hline & $\begin{array}{l}\text { Realiza resúmenes o sín- } \\
\text { tesis de lo abordado en } \\
\text { la vídeo-lección a ma- } \\
\text { nera de lo compilado. }\end{array}$ & $\begin{array}{l}\text { Se analiza si el docente concluye sus exposiciones re- } \\
\text { saltando los principales contenidos abordados, clarifi- } \\
\text { cando a modo de síntesis las ideas más importantes } \\
\text { desarrolladas. }\end{array}$ \\
\hline & $\begin{array}{l}\text { Menciona los puntos } \\
\text { que se verán en la pró- } \\
\text { xima lección. }\end{array}$ & $\begin{array}{l}\text { Se analiza si el docente ofrece un esquema claro sobre } \\
\text { los puntos que se desarrollarán en las diferentes leccio- } \\
\text { nes para que los usuarios puedan realizar el curso si- } \\
\text { guiendo una estructura fácil y organizada. }\end{array}$ \\
\hline
\end{tabular}


Tabla 3. Aspectos técnicos y de soporte narrativo audiovisual

$\begin{array}{lll}\text { Categorías Acciones del docente } & \text { Observaciones }\end{array}$

Se evidencian cambios de plano en la cámara.

Aspectos técnicos y de soporte narrativo audiovisual
La vídeo-lección cuenta con ayudas visuales dinámicas (gráficos en movimiento, infografías en movimiento.

Existe una variedad y relación de las imágenes con respecto de los contenidos mencionados por el docente a lo largo de la vídeo lección.
Se analizan los planos que se utilizan para poder valorar si existe una preocupación por la realización de los vídeos desde el punto de vista técnico, lo que podría ayudar a mantener la atención de los espectadores, o por el contrario disminuirla si fuesen demasiado monótonos o repetitivos.

Se tiene en consideración el uso de recursos visuales específicos para la vídeo-lección, como diapositivas generadas en Genial.ly, Prezzi, documentos gráficos, PDF, etc. así como, el uso de presentaciones dinámicas que incluyan gráficos e infografías que faciliten el proceso de aprendizaje.

Se estudia este ítem para saber si el material visual tiene relación con el planteamiento de los contenidos que se abordan en cada vídeo-lección, valorando la variedad de recursos visuales y su conexión o correspondencia con la temática del discurso que se expone.

\section{RESUlTADOS}

\section{a) Aspectos generales de los MOOC analizados}

Los tres MOOC analizados requieren de un registro previo en la plataforma correspondiente por parte del interesado para poder inscribirse, siendo la realización de los cursos de carácter gratuito en su totalidad. No obstante, en MiríadaX se indica el precio en los datos generales del curso, por un lado, para la obtención del certificado de superación $(40 €)$ y, por otro, para la obtención de la acreditación, que requiere la aprobación de un examen biométrico (140€). Dicha cantidad deberá ser abonada por el estudiante tras finalizar el curso en caso de solicitar alguna de las dos modalidades de certificación. La plataforma Edx también ofrece la posibilidad de añadir un certificado verificado de superación, indicando el precio en la información del MOOC (41€). Este tipo de datos no son facilitados en la información general del curso de la plataforma ECO, por lo que, al momento de inicio, el participante no dispone de información sobre la posibilidad de obtención de certificación. En este sentido, cabe destacar que en los MOOC ha de tenerse en cuenta que la obtención de certificados es uno más de los componentes motivadores de este tipo de enseñanza, por lo que se establecen como un elemento más que puede implicar al alumnado en la realización en estos cursos (Sánchez-Acosta et al., 2014). 
En cuanto a la duración de las vídeo-lecciones, el promedio oscila entre los que duran menos de 5 minutos y aquellos que duran entre 6 y 12 minutos. Esto se explicaría debido a la adaptación de los cursos a los hábitos digitales de los consumidores. La tendencia indica que se prefieren los vídeos cortos, principalmente por tres razones: disminución de la posibilidad de provocar cansancio, desconexión o aburrimiento en los espectadores; aumento de la capacidad para asimilar contenidos; y promoción del deseo de continuar viendo más (Gértrudix et al., 2017).

En lo referente a la tipología de las vídeo-lecciones, cabe destacar que la más común es la de tipo magistral, en la que los docentes emplean los recursos audiovisuales (principalmente presentaciones en Power Point) para exponer los contenidos desde un enfoque tradicional, ya que durante el vídeo el alumnado adquiere un rol pasivo en el que simplemente es receptor de la información, mientras que el profesorado, como experto en la materia, imparte la lección. No obstante, también se incluyen algunos vídeos en los que aparecen entrevistas a expertos en el área o bajo las modalidades Talking Head, en los que el profesorado explica los contenidos frente a la cámara, sin ningún tipo de apoyo visual, o Picture in picture, en los que se combinan las grabaciones de pantalla o las animaciones con la voz en off de los docentes explicando las imágenes. En este último caso, suelen ser contenidos para mostrar la estructura o funcionamiento de alguna plataforma, por ejemplo, en el módulo introductorio de ECO en el que se explicaba la interfaz y secciones del MOOC o vídeos realizados mediante algún software para crear animaciones como, por ejemplo, VideoScribe (Figura 1).

Figura 1. Ejemplos de la tipología de vídeo-lecciones
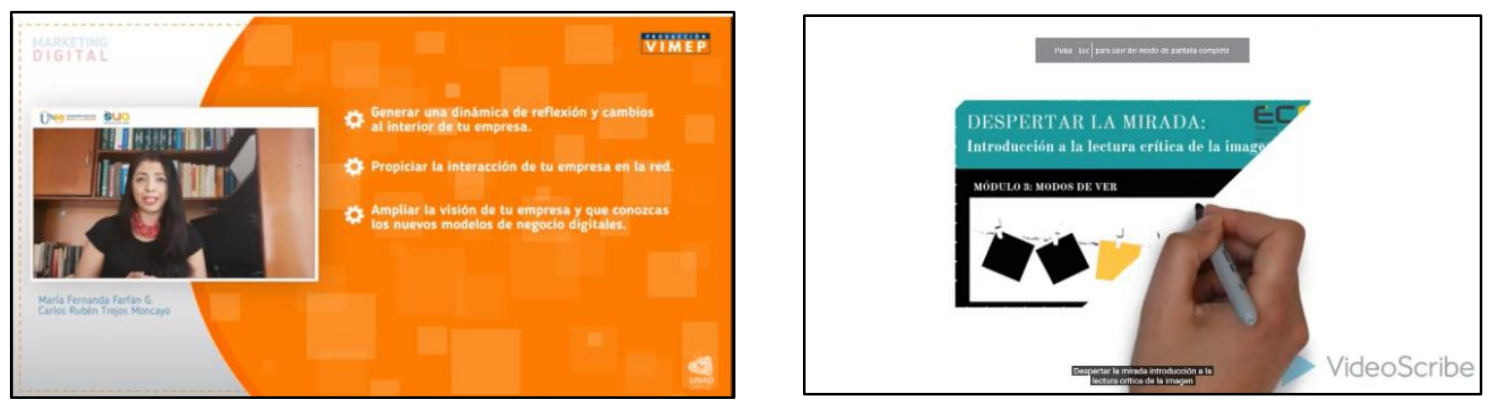

Las vídeo-lecciones, al ser grabadas y permitir la visualización de manera asincrónica, van siempre asociadas a la posibilidad de realizar aportes a los contenidos expuestos por los docentes a través de redes sociales y foros asociados al MOOC para debatir e intercambiar ideas y valoraciones con otros compañeros. 


\section{b) Inicio de las vídeo-lecciones}

En lo referente a los principios de engagement, en los comienzos de las vídeo-lecciones no se emplean estrategias para captar la atención de la audiencia de manera atrayente y llamativa. La estructura de los clips educativos tiende a desarrollarse mediante una perspectiva más tradicional, en la que se exponen los contenidos de manera explicativa comenzando generalmente por una introducción y presentación de los objetivos del módulo o la lección, continuando por el desarrollo teórico de los temas a tratar y finalizando con el planteamiento de conclusiones. Tal y como se evidencia en el instrumento utilizado, se podría realizar de manera más atractiva, planteando retos desde el inicio de las vídeo-lecciones o empezando la lección a través de preguntas, con el fin de despertar el interés del alumnado y fomentar la participación, creación y motivación. En la Tabla 4, se pueden apreciar las características de cada uno de los MOOC analizados en función de las categorías propuestas (Manotas-Salcedo et al., 2019).

Tabla 4. Inicio de las vídeo-lecciones en cada MOOC

\begin{tabular}{lccc}
\hline \multicolumn{1}{c}{ Acciones del docente } & MiríadaX & Edx & ECO \\
\hline $\begin{array}{l}\text { Comienza con una pregunta a la audiencia. } \\
\text { Plantea un reto a resolver durante la vídeo lección. }\end{array}$ & - & - & - \\
$\begin{array}{l}\text { Desglosa de manera clara y organizada los puntos } \\
\text { que se desarrollarán en la vídeo-lección. }\end{array}$ & $\checkmark$ & - & - \\
\hline
\end{tabular}

En lo relativo al MOOC ofertado en la plataforma de MiríadaX, en ninguno de los cuatro módulos que conformaban el curso se comenzaba realizando una pregunta al inicio de las sesiones, aunque sí se planteaban un reto inicial (a estilo de la superación de una prueba o ejercicio) que ha de ser evaluado en cada módulo para poder tener acceso a los siguientes. Asimismo, el docente desglosa el contenido que se desarrollará en la vídeo-lección a diferencia de los otros dos cursos analizados, en los que eso no se realiza.

$\mathrm{Al}$ igual que en el caso anterior, en el MOOC de la plataforma Edx no se comienzan los vídeos con preguntas, aunque sí se plantean cuestiones a lo largo de la vídeo-lección. Del mismo modo, aunque no se presentan retos para resolver en cada una de las lecciones se proponen ejercicios evaluadores en los diferentes módulos, presentando el objetivo de ofrecer conocimientos y motivar a los participantes hacia el emprendimiento como un reto de tipo genérico. Tanto los contenidos 
que se desarrollarán en cada una de las vídeo-lecciones como la estructura del curso no se especifica de forma clara.

Con respecto al MOOC de la plataforma ECO, la mayoría de los vídeos que componen los seis módulos del curso no han sido producidos específicamente para este, sino que son enlaces a vídeos externos de YouTube, por lo que no son vídeolecciones en las que las docentes del MOOC expliquen los contenidos (ello solo ocurre en tres vídeos). En los demás módulos los recursos principales eran otro tipo de contenidos creados por las profesoras como, por ejemplo, presentaciones en Prezi. Por este motivo, durante las vídeo-lecciones no se plantean retos al alumnado. No obstante, cada uno de los módulos se divide en tres secciones: "Detonante", "Ideas claves" y "Reto" y en esta tercera suele haber alguna propuesta de actividad para el alumnado, que si bien no se introduce en un vídeo aparece recogida en los "Comentarios del profesor" o en las presentaciones de Prezi. Asimismo, se incide y se anima al alumnado a compartir los resultados y fomentar la participación en redes sociales usando hashtags creados para el MOOC.

\section{b) Desarrollo de las vídeo-lecciones}

Durante las vídeo-lecciones, se evidenció que el discurso apelaba a lo estrictamente académico, en el que no se emplearon recursos como el humor, las ejemplificaciones con anécdotas personales, los cambios de ritmo o la introducción de puntos de giro o hechos inesperados (Tabla 5).

Tabla 5. Desarrollo de las vídeo-lecciones en cada MOOC

\begin{tabular}{lccc}
\hline \multicolumn{1}{c}{ Acciones del docente } & MiríadaX & Edx & ECO \\
\hline Usa giros de humor. & - & $\checkmark$ & - \\
Incluye anécdotas personales relacionadas con el & - & $\checkmark$ & - \\
tema de la vídeo-lección. & - & - & - \\
Utiliza vídeos en los que hay cambios de ritmo. & - & - & - \\
Introduce puntos de giro o hechos inesperados & - &
\end{tabular}

En el MOOC realizado en MiríadaX no se aprecian giros de humor ni cambios en la entonación que permitieran la enfatización de alguna frase o palabra durante el discurso, lo que provocaba cierta monotonía en el estilo de las vídeolecciones. Esta sensación se incrementaba debido a que durante los vídeos no existían cambios en el ritmo ni se empleaban efectos, iluminación o diferentes ángulos 
de toma. Asimismo, a pesar de que no se hizo alusión a ninguna anécdota personal, sí se usaron ejemplos de contextualización, aunque en ocasiones resultaban demasiado específicos al hacer referencia al ámbito latinoamericano y más concretamente a ejemplificaciones relativas a Colombia, lo que pudiera suponer una limitación debido a que las explicaciones no eran comparativas para que todos los participantes tuvieran una visión más global. De manera genérica, no se produjeron puntos de giro porque el formato habitual de evaluación siempre consistía en una prueba cronometrada de selección simple. No obstante, en el módulo tres la interacción con otros usuarios que también realizaban el MOOC fue un hecho inesperado, con respecto al desarrollo hasta el momento de la actividad y al diseño de los módulos previos.

En cambio, en el curso ofertado en Edx se utilizaron recursos humorísticos e incluso se incluyeron vídeos divertidos destinados a mantener el interés del receptor. Asimismo, se emplean recurrentemente anécdotas para ejemplificar y contextualizar las explicaciones. No existen cambios notables en el ritmo, a pesar de que, en algunos vídeos, los menos teóricos, sí varían los planos y la entonación. En este sentido, tampoco se introducen hechos inesperados porque todas las vídeo-lecciones siguen el mismo enfoque, aunque se podrían destacar las entrevistas y el cambio de escenario en algunos de los módulos.

Con respecto al MOOC de la plataforma ECO, en las vídeo-lecciones en las que aparecen las docentes no se emplea el humor en ningún momento. Se presenta la información de manera explicativa y descriptiva, con un tono meramente formal. En los vídeos enlazados a YouTube tampoco se suele apreciar ese tono humorístico, son más bien vídeos para reflexionar sobre los estereotipos y el poder de las imágenes, la publicidad, etc., pero desde un enfoque informativo a través de documentales, entrevistas a expertos o una recopilación de ejemplos descriptivos, todo con carácter reflexivo y estrictamente formal. En esta línea, en las vídeo-lecciones en las que aparecen las docentes no se exponen anécdotas personales para explicar los contenidos de los módulos. Normalmente, al tratarse de vídeos cortos se presenta la información de manera concisa, sin ejemplificar ni hacer alusión a experiencias personales y, en general, no existen cambios en el ritmo ni giros inesperados en las vídeo-lecciones.

\section{c) Cierre de las vídeo-lecciones}

Al finalizar las vídeo-lecciones, se emplean algunos recursos recopilatorios que sirven de conclusión a la temática abordada en cada uno de los módulos, lo que permite a los participantes comprender mejor los contenidos planteados. Sin embargo, 
hay otros factores que no se tienen en cuenta y que podrían enriquecer más esta fase final de las vídeo-lecciones (Tabla 6).

Tabla 6. Cierre de las vídeo-lecciones en cada MOOC

\begin{tabular}{lccc}
\hline \multicolumn{1}{c}{ Acciones del docente } & MiríadaX & Edx & ECO \\
\hline $\begin{array}{l}\text { Establece conclusiones de acuerdo con lo planteado } \\
\text { en el inicio de la vídeo-lección. }\end{array}$ & - & - & $\checkmark$ \\
$\begin{array}{l}\text { Realiza resúmenes o síntesis de lo abordado en la ví- } \\
\text { deo-lección a manera de compilado. }\end{array}$ & $\checkmark$ & - & - \\
$\begin{array}{l}\text { Menciona los puntos que se verán en la próxima lec- } \\
\text { ción. }\end{array}$ & - & - & - \\
\hline
\end{tabular}

En el MOOC de MiríadaX, no se realizaban conclusiones con respecto a lo planteado en los vídeos iniciales. Sin embargo, sí existe un material de descarga en archivo PDF, que aporta tanto este tipo de información como una síntesis de cada módulo. También cabe destacar que al finalizar los vídeos no se mencionan explícitamente por parte del docente los puntos que se desarrollarán en lecciones posteriores, aunque el alumnado podía consultar estos datos en la sección de la página web referente a la estructura del MOOC, en la que aparece disponible el título de cada módulo, así como el tiempo de duración, lo que contextualiza al usuario y le permite tener una perspectiva global de los contenidos que se abordarán.

El curso disponible en Edx no incluía una recopilación de lo tratado en el vídeo. Esto se debe a que muchos de ellos eran entrevistas y al terminar no se resumían los temas tratados. Como opción para poder sintetizar se ofrece la descripción del vídeo en la parte inferior. En cuanto a los puntos de las próximas lecciones, no se hace alusión por parte del docente a este tipo de información, ni siquiera en los vídeos introductorios se adelantan los contenidos que se abordarán en los módulos siguientes.

En el MOOC de ECO, las docentes establecían conclusiones a lo expuesto a lo largo de sus explicaciones en aquellos vídeos y recursos creados por ellas, aunque no realizaban una síntesis de los temas tratados anteriormente. Tampoco se concretaban los puntos a tratar en las próximas sesiones en ninguno de los vídeos. $\mathrm{Al}$ igual que anteriormente, esto se debe a que en muchos de los módulos los vídeos eran de terceros y no han sido diseñados específicamente para el MOOC. 


\section{d) Aspectos técnicos y visuales}

En este apartado se analizan los recursos audiovisuales y las técnicas empleadas en los diferentes MOOC (Tabla 7).

Tabla 7. Aspectos técnicos y visuales en cada MOOC

\begin{tabular}{lccc}
\hline & MiríadaX & Edx & ECO \\
\hline $\begin{array}{l}\text { Se evidencian cambios de plano en la cámara. } \\
\text { La vídeo-lección cuenta con ayudas visuales dinámi- } \\
\text { cas (gráficos e infografías en movimiento). }\end{array}$ & - & $\checkmark$ & $\checkmark$ \\
$\begin{array}{l}\text { Existe una variedad y relación de las imágenes con } \\
\text { respecto de los contenidos mencionados por el do- } \\
\text { cente a lo largo de la vídeo lección. }\end{array}$ & $\checkmark$ & $\checkmark$ & - \\
\hline
\end{tabular}

Con respecto al MOOC de MiríadaX, el plano de los vídeos siempre es frontal. El docente utiliza un único fondo de biblioteca y se limita a leer el texto de introducción, lo que puede incidir negativamente en la percepción del usuario con respecto a su dominio del tema. Todos los módulos están acompañados de recursos visuales, específicamente diapositivas generadas en Genial.ly con la explicación de cada uno de los puntos del módulo. De igual forma, existe un documento en PDF. Es importante mencionar que los materiales de apoyo, tales como la presentación y los PDF, no cuentan con un formato estandarizado más allá de la firma de pie con los logos de las instituciones que avalan el curso. Las presentaciones son dinámicas y cuentan con gráficos e infografías que facilitan el proceso de aprendizaje. Asimismo, todo el material visual y contenidos de apoyo guardan relación y están en el orden de cada planteamiento.

En cuanto al curso de la plataforma Edx, aunque durante los vídeos el docente generalmente habla de frente a la cámara, se evidencian cambios de planos y aparecen vídeos en los que interactúa con otras personas. Asimismo, todos los vídeos cuentan con recursos visuales de apoyo, destacando la voz en off con imágenes y vídeos referentes a los contenidos que se van presentando. También se muestran animaciones en algunas de las vídeo-lecciones, especialmente en los módulos iniciales. Cabe mencionar también que todo el material está conectado y tiene relación con los contenidos abordados.

En los vídeos en los que aparecen las docentes del MOOC disponible en la plataforma ECO, se evidencian cambios en los planos de cámara, combinando el 
primer plano, el plano medio corto y el plano medio. Las lecciones no suelen contar con ayudas visuales dinámicas integradas en el propio vídeo ni suelen incluir imágenes que acompañen al discurso. Tan solo en un recurso creado con Vídeo Scribe se cumple este aspecto, ya que las animaciones van cambiando en función de la lectura que realiza una profesora y que sirve de voz en off para el vídeo. No obstante, sí se incluyen en los diferentes módulos recursos complementarios como presentaciones en Prezi, vídeos de YouTube... También se suelen recomendar referencias bibliográficas y enlaces de interés como blogs, páginas web, etc.

\section{CONCLUSIONES}

El estudio de los MOOC permite conocer cómo se desarrollan los procesos de enseñanza-aprendizaje en entornos virtuales. Este análisis se ha centrado en la identificación de las características que pueden incidir en la motivación de los participantes de las vídeo-lecciones que conforman los tres cursos ofertados en las plataformas.

En este sentido, en relación con la primera categoría, el análisis muestra que al inicio de las vídeo-lecciones dos de los tres cursos no comienzan desglosando los contenidos que se desarrollarán o planteando retos a la audiencia y en ninguno de ellos se formulan preguntas a los estudiantes, lo que no promueve un rol activo por parte del alumnado desde el principio de las lecciones. Estos resultados apuntan a la necesidad de plantearse el nivel de engagement, por parte de los docentes, a la hora de diseñar este tipo de cursos, con el fin de fomentar la participación del alumnado. En cuanto al desarrollo y cierre de las vídeo-lecciones, cabe resaltar que en la mayoría de los vídeos analizados no se usan estrategias como los recursos humorísticos, las anécdotas o elementos sorprendentes, lo que pone de manifiesto que el planteamiento didáctico de los cursos adoptaba un enfoque estrictamente formal. Por último, en lo referente a los aspectos técnicos, los cursos están diseñados en un formato de producción audiovisual y materiales de apoyo poco atractivo y basado en las estrategias generales de difusión de contenidos en el entorno digital, con una baja integración de estrategias innovadoras tanto en el contenido como en la edición de los materiales, fomentando más la transmisión de información en lugar de la construcción de entornos de aprendizaje significativos (Manotas et al., 2018).

El uso de este tipo de recursos, de acuerdo con Wu y Gao (2018), es un factor diferencial y de éxito en los MOOC, permitiendo hacer más cercano e interesante el contenido y ayudando, además, a la transmisión de seguridad y 
confianza por parte del docente. Tal y como se plantea al inicio de la investigación, el diseño tradicional educativo de clases virtuales, presente en los sistemas analizados, manifiesta carencias en el uso de recursos narrativos o de elementos alternativos de gamificación/networking, lo que podría generar un bajo nivel de interés y compromiso por parte de los participantes.

Teniendo en cuenta que los MOOC han significado un importante aporte en la educación en línea al permitir el acceso libre y gratuito al conocimiento y, a su vez, al convertirse en un sistema de pruebas para innovar en modelos pedagógicos y desarrollar nuevas arquitecturas de la enseñanza, los resultados sugieren que es necesario plantearse el diseño instruccional y el uso de recursos educativos en los entornos virtuales en relación con la participación y la motivación del alumnado. Sus características de gratuidad, heterogeneidad, multiplicidad temática y life-long learning han cambiado por completo el escenario de dicho diseño, lo que permite incluir innovaciones y nuevas arquitecturas de enseñanza-aprendizaje. Serán, precisamente, estos nuevos recursos, los que permitirán reducir las tasas de abandono y las críticas que estas suponen desde la comunidad científica, para alcanzar una mayor efectividad y éxito de los MOOC.

En última instancia, se plantea como futura línea de investigación ampliar el análisis a más cursos MOOC con el objetivo de comprobar si se obtienen resultados semejantes en esta línea, así como, analizar la correlación entre aprendizaje, nivel de formación académica de los participantes, tendencias en comunicación y marketing digital. Ese análisis, centrado no ya en el diseño de los MOOC sino en su relación con el proceso de enseñanza-aprendizaje, nos puede aportar información sobre los parámetros en los que situar la educación en este tipo de recursos. Nos puede informar, por ejemplo, cómo gestionar el acercamiento por parte de personas con formación a los temas establecidos, manteniendo su interés y con el objetivo no solo de ampliar conocimientos sino de desarrollar su emprendimiento, por ejemplo, en el sector económico.

\section{REFERENCIAS}

Apple Inc. (2011). Challenge Based Learning: Take Action and Make a Difference. https://bit.ly/3lpwanE

Atapattu, T., y Falkner, K. (2017). Discourse Analysis to improve the effective engagement of MOOC videos. In 'LAK'17 Proceedings of the Seventh International Learning Analytics \& Knowledge Conference. Lak, Vancouver. https://doi.org/10.1145/3027385.3029470

Aguaded, I., y Medina-Salguero, R. (2015). Criterios de calidad para la valoración y gestión de MOOC. Revista Iberoamericana de educación a distancia, 18(2), 119-143. https://doi.org/10.5944/ried.18.2.13579 
Calvo-Salvador, M.A., Rodríguez-Hoyos, C., y Fernández-Díaz, E.M. (2016). ¿Cómo son los MOOC sobre educación? Un análisis de cursos de temática pedagógica que se ofertan en castellano. Digital Education Review, 29, 298-311. https://bit.ly/2FR2bo9

Castaño-Garrido, C., Maíz -Olazabalaga, I., y Garay-Ruiz, U. (2015). Design, Motivation and Performance in a Cooperative MOOC Course. Comunicar, 44, 19-26. https://doi.org/10.3916/C44-2015-02

Cabero, J., y Gisbert, M. (2005). La Formación en Internet. Guía para el diseño de materiales didácticos. Eduforma.

European Comission. (2019). The Digital Competence Framework 2.0. https://bit.ly/3hqASil

García, L. (2001). La educación a distancia: de la teoría a la práctica. Editorial Ariel. https://bit.ly/3hrLJIT

Gallego, M., y Gutiérrez, E. (2011). Analizar la comunicación mediada por ordenador para la mejora de los procesos de enseñanza-aprendizaje. Revista de currículum y formación del profesorado, 15(1), 23-39. https://bit.ly/2stz2VM.

Gértrudix, M., Rajas, M., Barrera, D., Bastida, M., y Soto, C. (2017). Realización de vídeo educativo: análisis de la producción audiovisual de los MOOC de URJCx. In J. Sierra-Sánchez (Ed.), Nuevas tecnologías audiovisuales para nuevas narrativas interactivas digitales en la era multidispositivo (pp. 289-302). McGraw Hill Education. https://bit.ly/3jauZ9v

Gómez-Galán, J., y Pérez-Parras, J. (2017). Luces y sombras del fenómeno MOOC: ¿representan una auténtica innovación educativa? Revista de pedagogía, 38(102), 237-259. https://bit.ly/2EsLwXo

Glance, D.G., Forsey, M., y Riley, M. (2013). The pedagogical foundations of massive open online courses. First Monday, 18(5-6), 1-29. https://doi.org/10.5210/fm.v18i5.4350

Hansch, A., Hillers, L., McConachie, K., Newman, C., Schildhauer, T., y Schmidt, P. (2015). Video and online learning: Critical reflections and findings from the field. HIIG Discussion Paper Series, (02), 1-34. https://doi.org/10.2139/ssrn.2577882

Igartua, J. (2006). Métodos cuantitativos de investigación en comunicación. Bosch.

Jansen, D., y Goes-Daniels, M. (2015). Institutional MOOC Strategies in Europe: Status Report Based on A Mapping Survey Conducted in October-December 2015. EADTU. https://bit.ly/3i $847 \mathrm{Hh}$

Kizilcec, R. F., Piech, C. y Schneider, E. (2013). Deconstructing Disengagement: Analyzing Learner Subpopulations in Massive Open Online Courses. In LAK '13: Proceedings of the Third International Conference on Learning Analytics and Knowledge. (pp. 170-179). ACM: Digital Library. https://doi.org/10.1145/2460296.2460330

Manotas-Salcedo, E., Pérez-Rodríguez, M.A., \& Contreras-Pulido, P. (2018). Análisis de Vídeolecciones en MOOC enfocados en la formación pedagógica de docentes en Educación Superior. Un estudio de caso. ReiDoCrea, 7, 248-259. https://bit.ly/3h8XpQQ

Manotas-Salcedo, E. (2019). Diseño de un modelo de producción de vídeo-lecciones basado en el Edu-entretenimiento para la formación pedagógica a través de MOOC. [Tesis doctoral]. Universidad de Huelva.

Manotas-Salcedo, E., Pérez-Rodríguez, M.A., \& Contreras-Pulido, P. (2019). Propuesta de diseño de instrumento para analizar vídeo-lecciones en MOOC. Alteridad. 14(1), 53-64. https://doi.org/10.17163/alt.v14n1.2019.04

Martín-Padilla, A.H. (2016, September 28). Plataformas MOOC. MOOCervatorio. Observatorio de investigación de sobre MOOC. https://bit.ly/2E5nz8I

Piñuel-Raigada, J.L. (2002). Epistemología, metodología y técnicas del análisis de contenido. Sociolinguistic Studies, 3(1), 1-42. https://bit.ly/34cG59M 
Mcgowan, A., y Hanna, P. (2016). How video lecture capture affects student engagement in a higher education computer programming course: A study of attendance, video viewing behaviours and student attitude. IEEE. https://doi.org/10.1109/echallenges.2015.7440966

Otzen, T. \& Manterola, C. (2017). Técnicas de muestreo sobre una población a estudio. Int. J. Morphol, 35(1), 227-232. http://dx.doi.org/10.4067/S0717-95022017000100037

Padilla-Hernández, A. L., Gámiz-Sánchez, V. M., \& Romero-López, M. A. (2019). Niveles de desarrollo de la competencia digital docente: una mirada a marcos recientes del ámbito internacional. Innoeduca: international journal of technology and educational innovation, 5(2), 140-150. https://doi.org/10.24310/innoeduca.2019.v5i2.5600

Piscitelli, A. (2015). ¿Está cambiando la tecnología la Universidad? Humanidades digitales y nuevas normales educativas. TELOS, 101, 12-22. https://bit.ly/32llyNU

Pérez-Rodríguez, M.A., \& Delgado-Ponce, Á. (2012). From digital and audiovisual competence to media competence: Dimensions and indicators. Comunicar, 39, 25-34.

https://doi.org/10.3916/C39-2012-02-02

Ramírez, M.S. y Ramírez, D.C. (2016). Inverted Learning Environments with Technology, Innovation and Flexibility: Student Experiences and Meanings. Journal of Information Technology Research, 9(1), 18-23. https://doi.org/10.4018/JITR.2016010102.

Reeves, T. (2006). How do you know they are learning? the importance of alignment in higher education. Learning Technology, 4(2), 294-309. https://doi.org/10.1504/IJLT.2006.011336

Romero-Rodríguez, L.M., de-Casas-Moreno, P., y Torres-Toukoumidis. Á. (2016). Dimensions and indicators of the information quality in digital media. Comunicar, 49, 91-100. https://doi.org/10.3916/C49-2016-09

Romero-Rodríguez, L.M., Ramírez-Montoya, M.S., y Valenzuela González, J.R. (2019). Gamification in MOOC: Engagement Application Test in Energy Sustainability Courses. IEEE Access, 7(1), 32093-32101. https://doi.org/10.1109/ACCESS.2019.2903230.

Sánchez-Acosta, E. Escribano-Otero, J Y Valderrama, F. (2014). Motivación en la educación masiva online Desarrollo y experimentación de un sistema de acreditaciones para los MOOC. Digital Education Review, 25, 18-35.

Teixes, F. (2014). Gamificación: fundamentos y aplicaciones (eBook). Editorial UOC.

Valenzuela-Arviszu, S., Castillo-Abdul, B., Mendoza-Domínguez, A., y Ramírez-Montoya, M. (2019). Innovación y Sustentabilidad Energética. Colaboración multidisciplinar, MOOC e investigación educativa. Narcea ediciones.

Vázquez-Cano, E., López-Meneses, E., y Sarasola, J. L. (2013). La Expansión del Conocimiento Abierto: Los MOOC. Octaedro.

White, S., Davis, H., Dickens, K.P., Leon-Urrutia, M., Sanchez-Vera, M.M. (2014). MOOC: What Motivates the Producers and Participants? In S. Zvacek, M. Restivo, J. Uhomoibhi y M. Helfert (eds) Computer Supported Education. CSEDU 2014. Communications in Computer and Information Science, (pp. 1-16). https://doi.org/10.1007/978-3-31925768-6 7

Wu H., Gao H. (2018) Chances and Challenges: How to Make a Successful MOOC. In G. Sun \& S. Liu (Eds.), Advanced Hybrid Information Processing. (pp 250-256) Springer. https://doi.org/10.1007/978-3-319-73317-3_30

Zheng, S., Rosson. M.B., Shih, P.C., y Carroll, J.M. (2015). Understanding student motivation, behaviors and perceptions in MOOC. In Proceedings of the 18th ACM Conference on Computer Supported Cooperative Work \& Social Computing, (pp.1882-1895). ACM. https://doi.org/10.1145/2675133.2675217 
Zhou, M. (2016). Chinese university 'students' acceptance of MOOC: A self-determination perspective. Computers \& Education, 92-93, 194-203.

https://doi.org/10.1016/j.compedu.2015.10.012

\section{BÁRBARA CASTILLO-ABDUL}

Investigadora Predoctoral del Departamento de Ciencias de la Comunicación y Sociología de la Universidad Rey Juan Carlos y Profesora Visitante del ESAI Business School, Universidad Espíritu Santo. Doctoranda en Comunicación por la Universidad de Huelva y la Universidad Rey Juan Carlos. Máster en Comunicación y Educación Audiovisual por la Universidad Internacional de Andalucía y la Universidad de Huelva. Editora Asociada de la Revista Index.Comunicación.

Barbara.castillo@urjc.es https://orcid.org/0000-0002-3711-1519

\section{MÓNICA BONILLA-DEL-RíO}

Doctoranda en el Programa Interuniversitario en Comunicación de las Universidades de Sevilla, Málaga, Huelva y Cádiz. Máster en Comunicación y Educación Audiovisual por la Universidad Internacional de Andalucía y la Universidad de Huelva.

monicabonilladelrio@gmail.com https://orcid.org/0000-0003-2476-8922

\section{SABINA Civila}

Doctoranda en el Programa Interuniversitario en Comunicación de las Universidades de Sevilla, Málaga, Huelva y Cádiz. Máster en Comunicación y Educación Audiovisual por la Universidad Internacional de Andalucía y la Universidad de Huelva.

sabicivila@gmail.com https://orcid.org/0000-0001-6059-9893

Castillo-Abdul, B., Bonilla-Del-Río, M. \& Civila, S. (2021). El engagement de participantes en MOOC (Massive Open Online Courses): Análisis del diseño instruccional y elementos alternativos. Bellaterra Journal of Teaching \& Learning Language \& Literature, 14(3), e944. https://doi.org/10.5565/rev/jtl3.944

Rebut / Recibido / Received / Reçu: 28-08-2021

Acceptat / Aceptado / Accepted / Accepté: 10-09-2021

https://revistes.uab.cat/jtl3/ 